\title{
Homoeologous copy-specific expression patterns of MADS- box genes for floral formation in allopolyploid wheat
}

\author{
Miku Tanaka ${ }^{1}$, Hiroko Tanaka ${ }^{1}$, Naoki Shitsukawa ${ }^{1}$, Satoshi Kitagawa ${ }^{1}$, \\ Shigeo Takumi ${ }^{2}$ and Koji Murai ${ }^{1 *}$ \\ ${ }^{1}$ Department of Bioscience, Fukui Prefectural University, 4-1-1 Matsuoka-kenjojima, \\ Eiheiji-cho, Yoshida-gun, Fukui 910-1195, Japan \\ ${ }^{2}$ Graduate School of Agricultural Science, Kobe University, \\ 1-1 Rokkodai, Nada, Kobe 657-8501, Japan
}

(Received 10 April 2015, accepted 23 July 2015; J-STAGE Advance published date: 25 November 2015)

The consensus model for floral organ formation in higher plants, the so-called ABCDE model, proposes that floral whorl-specific combinations of class A, B, C, D, and $\mathrm{E}$ genes specify floral organ identity. Class A, B, C, D and E genes encode MADS-box transcription factors; the single exception being the class A gene APETALA2. Bread wheat (Triticum aestivum) is a hexaploid species with a genome constitution AABBDD; the hexaploid originated from a cross between tetraploid T. turgidum (AABB) and diploid Aegilops tauschii (DD). Tetraploid wheat is thought to have originated from a cross between the diploid species $T$. urartu (AA) and Ae. speltoides (BB). Consequently, the hexaploid wheat genome contains triplicated homoeologous copies (homoeologs) of each gene derived from the different ancestral diploid species. In this study, we examined the expression patterns of homoeologs of class B, C and D MADS-box genes during floral development. For the class B gene wheat PISTILLATA2 (WPI2), the homoeologs from the $\mathrm{A}$ and $\mathrm{D}$ genomes were expressed, while expression of the $\mathrm{B}$ genome homoeolog was suppressed. For the class C gene wheat AGAMOUS1 (WAG1), the homoeologs on the A and B genomes were expressed, while expression of the D genome homoeolog was suppressed. For the class D gene wheat SEEDSTICK (WSTK), the B genome homoeolog was preferentially expressed. These differential patterns of homoeolog expression were consistently observed among different hexaploid wheat varieties and synthetic hexaploid wheat lines developed by artificial crosses between tetraploid wheat and Ae. tauschii. These results suggest that homoeolog-specific regulation of the floral MADS-box genes occurs in allopolyploid wheat.

Key words: ABCDE model, homoeologous gene, homoeolog, MADS-box gene, polyploidy

\section{INTRODUCTION}

The ABCDE model of flower development proposes that floral organ identity is mediated by five classes of homeotic genes, named A, B, C, D and E (Rijpkema et al., 2010). According to this model, class A and E protein complexes induce sepal formation as the ground-state floral organs in the first floral whorl; class A, B and E protein complexes specify petals in the second whorl; class B, $\mathrm{C}$ and $\mathrm{E}$ protein complexes specify stamens in the third whorl; and class $\mathrm{C}$ and $\mathrm{E}$ protein complexes specify car-

\footnotetext{
Edited by Hiro-Yuki Hirano

* Corresponding author. E-mail: murai@fpu.ac.jp

DOI: http://doi.org/10.1266/ggs.15-00029
}

pels in the fourth whorl. The ABCDE homeotic genes in Arabidopsis encode MADS-box transcription factors, the exception being the class A gene, APETALA2 (AP2). The Arabidopsis genome includes the class A MADS-box gene AP1 (Mandel et al., 1992), the class B genes AP3 and PISTILLATA (PI) (Jack et al., 1992; Goto and Meyerowits, 1994), the class $\mathrm{C}$ gene AGAMOUS (AG) (Yanofsky et al., 1990), the class D genes SEEDSTICK (STK), SHATTERPROOF1 (SHP1) and SHP2 (Favaro et al., 2003; Pinyopich et al., 2003), and the class E genes SEPALLATA1 (SEP1), SEP2, SEP3 and SEP4. The class $\mathrm{E}$ genes show partially redundant functions in determination of sepal, petal, stamen and carpel identities (Pelaz et al., 2000; Ditta et al., 2004).

The ABCDE model applies to wheat flower develop- 
ment (Murai, 2013). The wheat inflorescence (spike or ear) is composed of spikelets that, in turn, are composed of florets. In each floret, the reproductive organs are enveloped by two leaf-like structures, the lemma and the palea. The lemma is a bract and the palea is a prophyll, the first leaf formed by the axillary meristem (Shitsukawa et al., 2007). An individual wheat flower contains one pistil, three stamens and two lodicules that are modified petals. Thus, the wheat floral organ has a palea, lodicules, stamens and a pistil in whorls $1,2,3$, and 4 , respectively.

Wheat FRUITFULL2 (WFUL2) is a candidate wheat class A gene (Kinjo et al., 2012). WPI1 (wheat PISTILLATA1) and WPI2 are class B genes (Hama et al., 2004). A phylogenetic analysis using deduced amino acid sequences indicated that WPI1 and WPI2 are orthologs of the rice PI-type genes OsMADS4 and OsMADS2, respectively (Murai, 2013). WPI1 and WPI2 have also been called TaPI-1 and TaPI-2/TaAGL26, respectively (Zhao, T. et al., 2006; Paolacci et al., 2007). The AG orthologs of wheat, WAG1 (wheat AGAMOUS1) and WAG2, were identified as class $\mathrm{C}$ genes (Meguro et al., 2003; Hirabayashi and Murai, 2009). A phylogenetic analysis using the deduced amino acid sequences showed that WAG1 and WAG2 are orthologs of the rice $A G$-type genes, OsMADS58 and OsMADS3, respectively (Murai, 2013). WAG1 and WAG2 are also known as TaAG-1 and TaAG-2/TaAGL39, respectively (Zhao, T. et al., 2006; Paolacci et al., 2007). Two studies in wheat identified five genes, TaAGL2, TaAGL9, TaAGL31, TaAG-3A and TaAG-3B, as candidate orthologs of the rice class $\mathrm{D}$ gene OsMADS13 (Zhao, T. et al., 2006; Paolacci et al., 2007). Sequence analyses showed that $T a A G-3 A$ is identical with TaAGL9, and TaAG-3B is identical with TaAGL2. Furthermore, TaAGL2and TaAGL9 show very high sequence similarity. As OsMADS13 is orthologous to Arabidopsis $S T K$, these wheat orthologs have been renamed as WSTK (wheat SEEDSTICK) (Yamada et al., 2009).

Bread wheat is an allohexaploid with the genomic constitution AABBDD in which each genome originated from a different ancestral species. The A genome is believed to be derived from Triticum urartu, the B genome from Aegilops speltoides or another species in the Sitopsis section, and the $\mathrm{D}$ genome from Ae. tauschii (Feldman, 2001). Allopolyploidization leads to the generation of duplicated homoeologous gene-copies (homoeologs) and, consequently, the hexaploid wheat genome contains triplicated homoeologs derived from the three ancestral diploid species. With regard to the class E SEP-like genes, two MADS-box genes, WSEP (wheat SEPALLATA) and WLHS1 (wheat LEAFY HULL STERILE 1) have been identified in wheat (Shitsukawa et al., 2007). Analyses of gene structure, expression patterns and protein functions showed no evolutionary changes in the WSEP homoeologs. In contrast, the three WLHS1 homoeologs show genetic and epigenetic alterations. The A genome homoeolog (WLHS1-A) has a large deletion in the coding region, and does not have a function in floral development. WLHS1-B and WLHS1-D, located in the B and $\mathrm{D}$ genomes, respectively, have the complete MADSbox gene structure; however, WLHS1-B is effectively silenced by epigenetic modification. The diversification that has occurred among WLHS1 homoeologs suggests that homoeologs of other homeotic MADS-box genes may also be differentially regulated in wheat spike formation. The floral homeotic MADS domain proteins have been proposed to interact in the floral tissue in the "floral quartet" model, in which a tetramer of MADS domain proteins functions in specification of floral organ identity (Smaczniak et al., 2012). Complex interactions among the homoeologous copies are probably associated with morphological and physiological diversification of floral development among species with different ploidy levels. In the present study, we examined homoeologspecific expression patterns of the class B, C and D genes, WPI2, WAG1 and WSTK, respectively, in tetraploid and hexaploid wheat. Our results suggest that homoeologspecific expression patterns of floral MADS-box genes are determined and controlled by an as yet uncharacterized regulatory system that developed during allopolyploidization.

\section{MATERIALS AND METHODS}

Plant materials One wild tetraploid species (T. turgidum ssp. dicoccoides), five cultivated tetraploid species $(T$. turgidum ssp. durum and four ssp. dicoccon), four hexaploid bread wheat cultivars (T. aestivum ssp. aestivum), and four hexaploid species ( $T$. aestivum ssp. sphaerococcum, ssp. compactum, ssp. spelta and ssp. macha) were used in this study (Table 1). These materials were obtained from NBRP-KOMUGI (National Bioresource Project-KOMUGI, Japan; http://shigen.lab.nig.ac.jp/wheat/komugi). Four synthetic hexaploid wheat lines were also used. Two were generated previously by crossing $T$. turgidum ssp. dicoccoides or ssp. dicoccon with Ae. tauschii; they were provided by NBRP-KOMUGI. Two synthetic hexaploid lines ( $F_{4}$ generation) were generated recently from crosses between T. turgidum ssp. durum cv. Langdon and Ae. tauschii (Kajimura et al., 2011). These synthetic hexaploid lines have the normal chromosome set $(2 n=42)$ and grow normally. Wheat plants were grown in the experimental field at Fukui Prefectural University.

Identification of homoeologs for class $B, C$, and $D$ MADS-box genes The sequences of class B, C, D MADS-box genes were obtained by a database search of DDBJ (DNA Data Bank of Japan) and NBRP-KOMUGI websites. The chromosomal location of each gene was determined by PCR analysis with homoeolog-specific 
Table 1. Plant materials

\begin{tabular}{|c|c|c|c|c|}
\hline \multicolumn{5}{|c|}{ Tetraploid (Wild) } \\
\hline No. & Species & NBRP No. & Country & Genome \\
\hline 1 & T. turgidum ssp. dicoccoides var. kotschyanum & KU-108-2 & Syria & $\mathrm{AABB}$ \\
\hline \multicolumn{5}{|c|}{ Tetraploid (Cultivated) } \\
\hline No. & Species & NBRP No. & Country & Genome \\
\hline 1 & T. turgidum ssp. durum cv. Langdon & LPGKU2196 & unknown & $\mathrm{AABB}$ \\
\hline 2 & T. turgidum ssp. dicoccon var. farrum & KU-113 & unknown & $\mathrm{AABB}$ \\
\hline 3 & T. turgidum ssp. dicoccon var. atratum & KU-115 & unknown & $\mathrm{AABB}$ \\
\hline 4 & T. turgidum ssp. dicoccon var. liguliforme & KU-111 & unknown & $\mathrm{AABB}$ \\
\hline 5 & T. turgidum ssp. dicoccon var. farrum & KU-123 & unknown & $\mathrm{AABB}$ \\
\hline
\end{tabular}

Hexaploid (T. aestivum ssp. aestivum: Bread wheat)

\begin{tabular}{clcccc}
\hline No. & & Variety & NBRP No. & Country & Genome \\
\hline 1 & Chinese Spring & KU-184-1 & China & AABBDD \\
2 & Nanbukomugi & KT020-617 & Japan & AABBDD \\
3 & ND & KU-11809 & Greece & AABBDD \\
4 & ND & KU-1005 & Spain & AABBDD \\
\hline
\end{tabular}

Hexaploid (Excepting Bread wheat)

\begin{tabular}{clccc}
\hline No. & \multicolumn{1}{c}{ Species } & NBRP No. & Country & Genome \\
\hline 1 & T. aestivum ssp. sphaerococcum var. rotundatum & $\mathrm{Sphr}^{1)}$ & unknown & AABBDD \\
2 & T. aestivum ssp. compactum cv. No. 44 & Cmp $^{1)}$ & unknown & AABBDD \\
3 & T. aestivum ssp. spelta var. duhamelianum & Splt $^{1)}$ & unknown & AABBDD \\
4 & T. aestivum ssp. macha var. subletschchumicum & $\mathrm{Mch}^{1)}$ & unknown & AABBDD \\
\hline
\end{tabular}

Synthetic hexaploid

\begin{tabular}{|c|c|c|c|}
\hline No. & Combination & NBRP No. & Genome \\
\hline 1 & $\begin{aligned} \text { T. turgidum } & \text { ssp. dicoccoides var. spontaneonigrum KU-109 } \\
& \times \text { Ae. tauschii } \text { ssp. strangulata KU-2074 }\end{aligned}$ & KU-221-12 & AABBDD \\
\hline 2 & T. turgidum ssp. dicoccon cv. Vernal $\times$ Ae. tauschii & KU-222 & AABBDD \\
\hline 3 & T. turgidum ssp. durum cv. Langdon $\times$ Ae. tauschii cv. PI476874 & $6256^{2)}$ & AABBDD \\
\hline 4 & T. turgidum ssp. durum cv. Langdon $\times$ Ae. tauschii cv. CGN10768 & $6277^{2)}$ & AABBDD \\
\hline
\end{tabular}

primer sets (described below) (Table 2). These analyses were performed using nulli-tetrasomic lines of bread wheat cultivar 'Chinese Spring' in which a pair of A, B, or $\mathrm{D}$ genome chromosomes was substituted by a pair of homoeologous chromosomes. The nulli-tetrasomic lines were obtained from NBRP-KOMUGI. We describe homoeologs located on chromosomes A, B and D as 'gene name-A, gene name-B and gene name-D', respectively. The accession numbers of homoeologs for class B, C, D MADS-box genes (WPI2, WAG1 and WSTK) are shown in Table 3. Based on single nucleotide polymorphisms and/ or nucleotide indels, we designed homoeolog-specific primers for WPI2, WAG1 and WSTK (Supplementary Figs. S1, S2 and S3). The sequences of the primer sets used in this study are listed in Table 2. Using the homoeolog-specific PCR primer sets, homoeolog-specific real-time PCR analyses were carried out to investigate differences in expression levels of the MADS-box homoeologs.

Real-time PCR analysis Expression analyses were performed using RNAs extracted from young spikes at the 
Table 2. Sequences of primer sets used for real-time PCR analyses

\begin{tabular}{|c|c|c|c|c|}
\hline Gene & Primer name & Sequences $\left(5^{\prime}-3^{\prime}\right)$ & $\begin{array}{l}\text { Product } \\
\text { size (bp) }\end{array}$ & $\begin{array}{c}\text { Annealing } \\
\text { temperature }\left({ }^{\circ} \mathrm{C}\right)\end{array}$ \\
\hline \multirow[t]{2}{*}{ Actin } & actin361-L & TATGCCAGCGGTCGAACAAC & 361 & 65 \\
\hline & actin361-R & GGAACAGCACCTCAGGGCAC & & \\
\hline \multirow[t]{2}{*}{ WPI2-A } & WPI2-AspeL & CATTTTGCCAGTTGTATCCTTCA & 240 & 65 \\
\hline & WPI2-AspeR & CTATTCACCGTCCGGCAAC & & \\
\hline \multirow[t]{2}{*}{ WPI2-B } & WPI2-BspeL & CCTTCGTTTGTTATCTGTTTGGAC & 225 & 70 \\
\hline & WPI2-BspeR & ACTATTCACCGTCCAGCAATTGT & & \\
\hline \multirow[t]{2}{*}{ WPI2-D } & WPI2-DspeL & CTAGGCCACCGACTCCACA & 305 & 70 \\
\hline & WPI2-DspeR & TATTCACCGTCCGGCAATC & & \\
\hline \multirow[t]{2}{*}{ WAG1-A } & WAG1-AspeL & TCAACTTCATGCAGCAGCAC & 213 & 70 \\
\hline & WAG1-AspeR & ATAGGATAAAGTCGATTGTGCGC & & \\
\hline \multirow[t]{2}{*}{ WAG1-B } & WAG1-BspeL & TCGGGCTCAGGATCGC & 358 & 70 \\
\hline & WAG1-BspeR & TGGAGTTCTGCAAGGTGGTTATT & & \\
\hline \multirow[t]{2}{*}{ WAG1-D } & WAG1-DspeL & CGCATCGAGAACACGACC & 366 & 70 \\
\hline & WAG1-DspeR & CAGCCTTCCCTCCAGCTGT & & \\
\hline \multirow[t]{2}{*}{ WSTK-A } & WSTK-AspeL & AGGGAGATAGAGCTTCAGAGTGACAA & 230 & 68 \\
\hline & WSTK-AspeR & TGAAGCGAGGCCTGA & & \\
\hline \multirow[t]{2}{*}{ WSTK-B } & WSTK-BspeL & GCGGAGGAGGAGCAGCA & 166 & 72 \\
\hline & WSTK-BspeR & CCTGAGCCTGAGCCTGAGTCC & & \\
\hline \multirow[t]{2}{*}{ WSTK-D } & WSTK-DspeL & AAAGGGAGATAGAGCTTCAGAGTGACAG & 228 & 72 \\
\hline & WSTK-DspeR & GCTGAAGCGAGGCCTGAGCC & & \\
\hline
\end{tabular}

Table 3. Three homoeologs of WPI2, WAG1 and WSTK

\begin{tabular}{|c|c|c|c|c|}
\hline Gene & Homoeologs & Accession no. & Synonym/Clone & Reference \\
\hline \multirow[t]{6}{*}{ WPI2 } & $W P I 2-A$ & CJ683155/CJ576494 & whpc11d $19^{1)}$ & Mochida et al. 2006 \\
\hline & WPI2-B & AB107992 & WPI2 & Hama et al. 2004 \\
\hline & & AM502881 & TaPI-2 (WM15) & Paolacci et al. 2007 \\
\hline & & CJ685552/CJ578977 & whpc $20 \mathrm{k} 17^{1)}$ & Mochida et al. 2006 \\
\hline & WPI2-D & DQ512343 & TaAGL26 & Zhao et al. 2006 \\
\hline & & CJ660742/CJ553194 & whhg5o03 ${ }^{1)}$ & Mochida et al. 2006 \\
\hline \multirow[t]{5}{*}{ WAG1 } & $W A G 1-A$ & CJ686121/CJ579589 & whpc4g11 ${ }^{1)}$ & Mochida et al. 2006 \\
\hline & WAG1-B & AB084577 & $W A G$ & Meguro et al. 2003 \\
\hline & & CJ682811/CJ576124 & whok9l15 $5^{1)}$ & Mochida et al. 2006 \\
\hline & WAG1-D & AM502863 & $T a A G-1(W M 2)$ & Paolacci et al. 2007 \\
\hline & & CJ677819/CJ570945 & whok $10 \mathrm{e} 04^{1)}$ & Mochida et al. 2006 \\
\hline \multirow[t]{8}{*}{ WSTK } & WSTK-A & DQ512337 & TaAGL2 & Zhao et al. 2006 \\
\hline & & AM502865 & $T a A G-3 B(W M 3 B)$ & Paolacci et al. 2007 \\
\hline & & CJ676578/CJ569669 & whms5h04 ${ }^{1)}$ & Mochida et al. 2006 \\
\hline & $W S T K-B$ & BJ260748/BJ266438 & whh26k17 1 () & Mochida et al. 2006 \\
\hline & WSTK-D & DQ512364 & TaAGL9 & Zhao et al. 2006 \\
\hline & & DQ512349 & TaAGL31 & Zhao et al. 2006 \\
\hline & & AM502864 & $T a A G-3 A(W M 3 A)$ & Paolacci et al. 2007 \\
\hline & & CJ629480/CJ520771 & whes $19 \mathrm{k} 15^{1)}$ & Mochida et al. 2006 \\
\hline
\end{tabular}

1) EST clones. The sequence information is shown by 5 -sequence accession no./3'sequence accession no. The connecting (contig) sequences are considered to be the full-length cDNA sequences and used in this study. 
floral organ developmental stage (3-10 $\mathrm{mm}$ in length), and from stamens and pistils of heading stage plants grown in the field. Samples were collected from three to five individuals, and each assay was performed with three replications. Total RNAs were extracted using ISOGEN (Nippon-gene, Japan) and cDNAs were subsequently synthesized with oligo-dT primer in accordance with the protocol for the Ready-To-Go T-Primed First-Strand Kit (GE Healthcare Life Sciences). Real-time RT-PCR analyses were performed using a LightCycler 2.0 (Roche Diagnostics $\mathrm{GmbH})$ with homoeolog-specific primer sets for WPI2, WAG1 and WSTK (Table 2). The relative quantities of transcripts were normalized against SYBR Green fluorescence of the Actin gene. For comparison of expression levels among homoeologs, we examined the amplification efficiency of each primer set using 10 -fold dilutions $(\times 1$, $\times 10, \times 100, \times 1,000, \times 10,000)$ of the gene-specific plasmids and compared their relative amplification efficiencies (Supplementary Fig. S4). The gene expression level of each homoeolog was standardized based on the amplification efficiency of its primer set and these revised levels of expression were used for comparison of homoeologs. Differences in gene expression levels were analyzed statistically using $t$-tests. Possible nucleotide polymorphisms within the homoeolog-specific primer sites of the varieties were not examined in the present study.

\section{RESULTS}

Identification of homoeologs for class B, C, D MADS-box genes in hexaploid wheat The $P I$-type genes WPI1 (wheat PISTILLATA1) and WPI2 have been identified as class B genes in wheat (Hama et al., 2004). WPI1 and WPI2 are also called TaPI-1 and TaPI2/TaAGL26, respectively (Paolacci et al., 2007; Zhao, T. et al., 2006). Here, sequence and PCR analyses of genomic DNA from nulli-tetrasomics indicated that TaPI2 was identical with WPI2, and that WPI2/TaPI-2 and TaAGL26 were homoeologous and located on chromosomes $3 \mathrm{~B}$ and $3 \mathrm{D}$, respectively (Fig. 1A, Table 3). In this report, we call these genes WPI2-B and WPI2-D, respectively. A third homoeolog, WPI2-A, was detected in the DDBJ database as an EST clone (whpc11d19; Mochida et al., 2006). The cDNA coding sequences of the homoeologs have eight single nucleotide polymorphisms (SNPs) (Supplementary Fig. S1), and three amino acid alterations (one located in the I-region and two in the Kdomain) were identified in the deduced polypeptide sequences of their proteins (Supplementary Fig. S5).

The wheat $A G$ orthologs WAG1 (wheat AGAMOUS1) and WAG2 were identified as class $\mathrm{C}$ genes (Meguro et al., 2003; Hirabayashi and Murai, 2009). WAG1 and WAG2 are also known as TaAG-1 and TaAG-2/TaAGL39, respectively (Zhao, T. et al., 2006; Paolacci et al., 2007). Sequence and PCR analyses indicated that WAG1 and
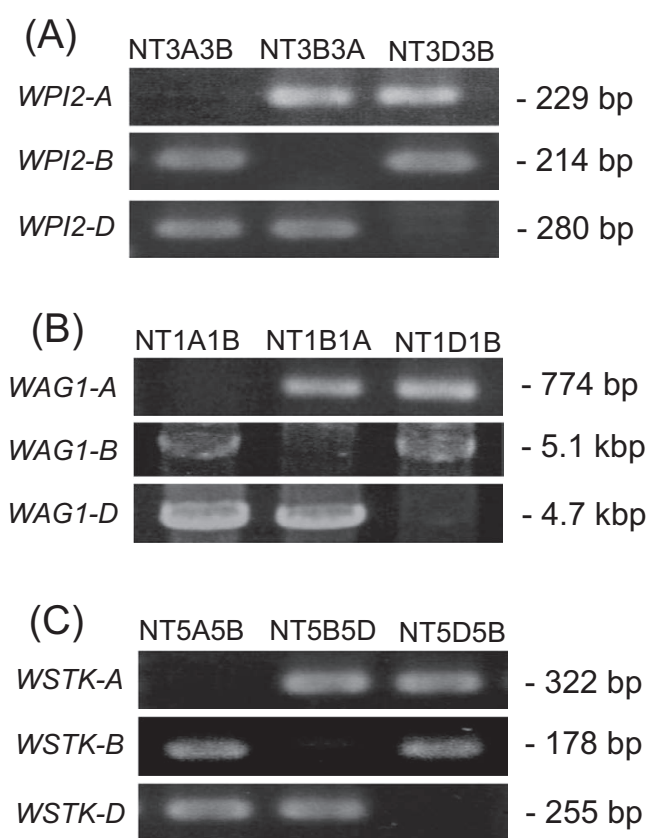

Fig. 1. PCR assay using genomic DNA of nulli-tetrasomics as the template and homoeolog-specific primer sets to determine the chromosomal locations of class B, C and D MADS-box gene homoeologs. (A) The WPI2-A, WPI2-B and WPI2-D homoeologs are located on chromosomes $3 \mathrm{~A}, 3 \mathrm{~B}$ and $3 \mathrm{D}$, respectively. (B) The WAG1-A, WAG1-B and WAG1-D homoeologs are located on chromosomes $1 \mathrm{~A}, 1 \mathrm{~B}$ and $1 \mathrm{D}$, respectively. (C) The WSTK-A, WSTK-B and WSTK-D homoeologs are located on chromosomes $5 \mathrm{~A}, 5 \mathrm{~B}$ and 5D, respectively. Homoeolog-specific primer sets for each MADS-box gene are shown in Table 2. The accession numbers of the homoeologs are shown in Table 3.

TaAG-1 were homoeologs and were located on chromosome $1 \mathrm{~B}$ and $1 \mathrm{D}$, respectively (Fig. $1 \mathrm{~B}$, Table 3 ); we term these genes WAG1-B and WAG1-D in this report. In the DDBJ database, we identified WAG1-A (whpc4g11) (Mochida et al., 2006), which is located on chromosome 1A. The homoeologous genes had nine SNPs in their cDNA coding sequences (Supplementary Fig. S2), and three amino acid alterations in the C-region were found in the deduced polypeptide sequences (Supplementary Fig. S6). Some of the AGAMOUS homologs had an Nregion upstream of the MADS-box region. Since it is not certain that WAG1 has an N-region, we considered the ATG codon immediately preceding the MADS-domain to be the initiation codon.

The genes TaAGL2, TaAGL9, TaAGL31, TaAG-3A and TaAG-3B have been identified as candidate orthologs of the rice class D gene OsMADS13 (Zhao, T. et al., 2006; Paolacci et al., 2007). Sequence analyses showed that TaAG-3A was identical to TaAGL9, and that TaAG-3B was identical to TaAGL2. Contrary to Zhao, T. et al. (2006), our sequence analysis indicated that TaAGL31 was identical to TaAGL9. In the wheat EST database in DDBJ, we identified another WSTK homolog, clone whh26k17 (Mochida et al., 2006). Our PCR assay indi- 
cated that TaAGL2, whh26k17 and TaAGL9 corresponded to WSTK-A, WSTK-B and WSTK-D, respectively (Fig. 1C, Table 3). The homoeologs have 16 SNPs and six base insertion/deletions in the cDNA coding sequences (Supplementary Fig. S3), and ten amino acid alterations and two amino acid insertion/deletions in the deduced polypeptide sequences of their proteins (Supplementary Fig. S7). There was a single amino acid alteration in the K-box domains and nine in the C-regions of the homoeologs, suggesting functional divergence among the three WSTK homoeologs.

Expression pattern of class B gene WPI2 in hexaploid and tetraploid wheat The levels of expression of WPI2 homoeologs were compared in hexaploid wheat varieties (Fig. 2). The A genome and D genome homoeologs, WPI2-A and WPI2-D, were expressed in young spikes and stamens (Fig. 2, A and B). In contrast,
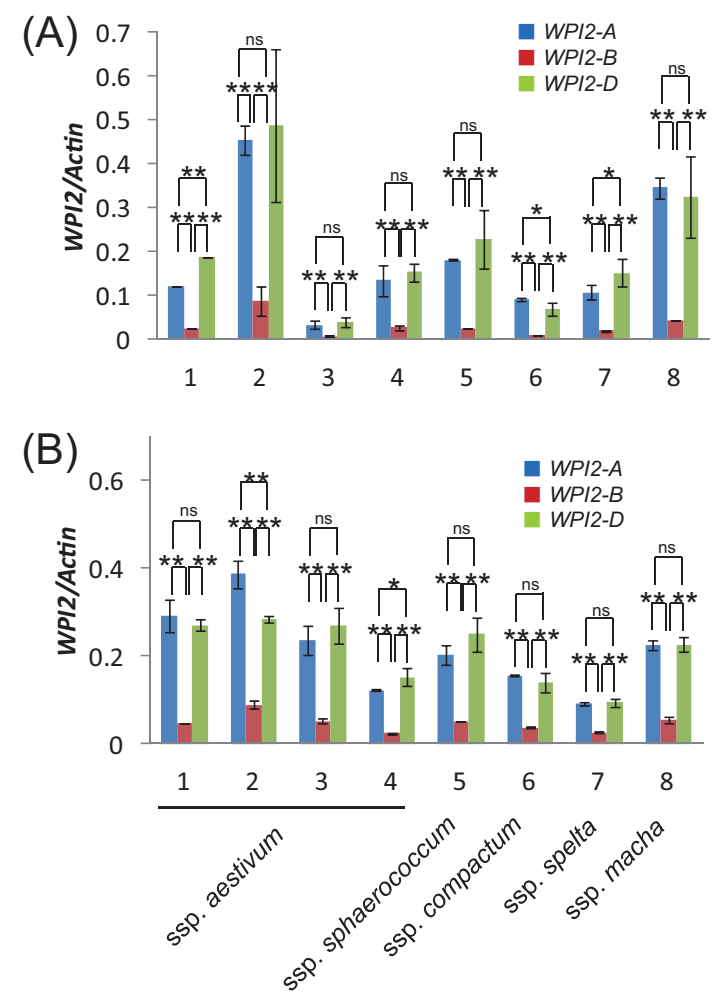

Fig. 2. Expression patterns of three WPI2 homoeologs examined by real-time PCR in hexaploid wheat using homoeologspecific primers for WPI2-A, WPI2-B and WPI2-D. Actin was used as the endogenous control. Total RNAs were isolated from (A) young spikes (3 to $10 \mathrm{~mm}$ in length) and (B) stamens of hexaploid species: (1) T. aestivum ssp. aestivum cv. Chinese Spring; (2) T. aestivum ssp. aestivum cv. Nanbukomugi; (3) T. aestivum ssp. aestivum (KU-11809); (4) T. aestivum ssp. aestivum (KU-1005); (5) T. aestivum ssp. sphaerococcum; (6) T. aestivum ssp. compactum; (7) T. aestivum ssp. spelta; (8) T. aestivum ssp. macha. Data represent means \pm standard errors from three replicate experiments. "ns" indicates no significant difference; asterisk indicates a significant difference in a $t$-test $(*, P<0.05$; **, $P<0.01)$. the B genome homoeolog WPI2-B was significantly downregulated in all varieties, suggesting that suppression of WPI2-B expression was a common feature of hexaploid wheat. The suppression of WPI2-B was also observed in the stamens of tetraploid wheat varieties (Fig. 3B). Interestingly, in young spikes, WPI2-B was not downregulated, and showed higher expression than WPI2-A (Fig. 3A). These observations indicated that the upregulation of WPI2- $A$ and down-regulation of WPI2-B in young spikes occurred during the allohexaploidization process in progeny of crosses of tetraploid wheat and the diploid D genome donor Ae. tauschii.

Expression pattern of class $C$ gene WAG1 in hexaploid and tetraploid wheat The levels of expression of WAG1 homoeologs were compared in hexaploid wheat varieties (Fig. 4). In young spikes from hexaploid wheat lines, WAG1-A was expressed but WAG1-B and WAG1-D were significantly down-regulated (Fig. 4A). In stamens and pistils, WAG1-B was up-regulated compared
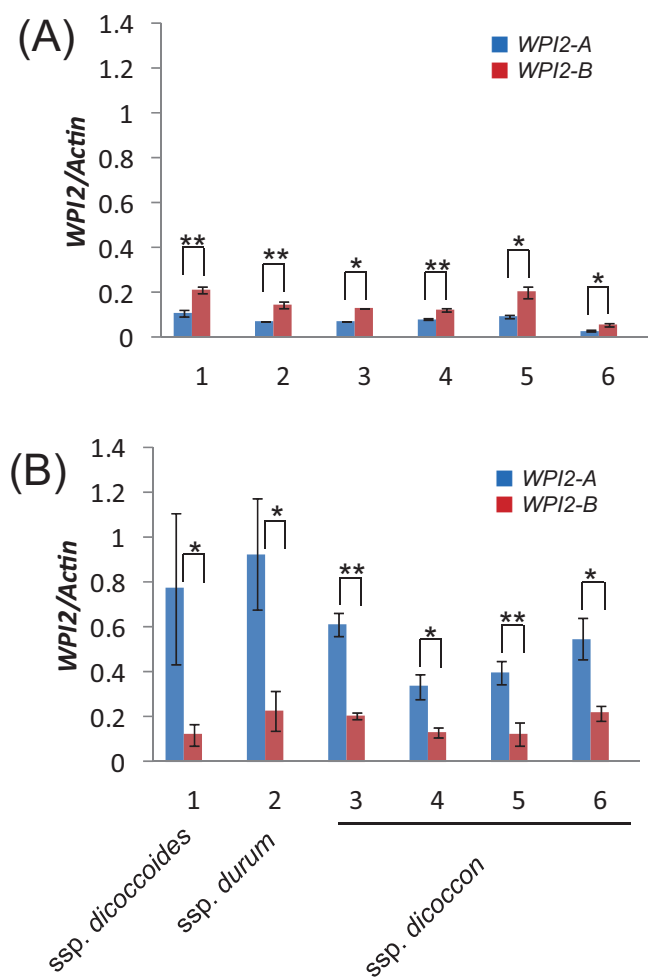

Fig. 3. Expression patterns of three WPI2 homoeologs examined by real-time PCR in tetraploid wheat using homoeologspecific primers for WPI2-A and WPI2-B. Actin was used as the endogenous control. Total RNAs were isolated from (A) young spikes (3 to $10 \mathrm{~mm}$ in length) and (B) stamens of tetraploid species: (1) T. turgidum ssp. dicoccoides; (2) T. turgidum ssp. durum; (3) T. turgidum ssp. dicoccon (KU-113); (4) T. turgidum ssp. dicoccon (KU-115); (5) T. turgidum ssp. dicoccon (KU-111); (6) T. turgidum ssp. dicoccon (KU-123). Data represent means \pm standard errors from three replicate experiments. "ns" indicates no significant difference. Asterisk indicates a significant difference in a $t$-test $(*, P<0.05$; **, $P<0.01)$. 

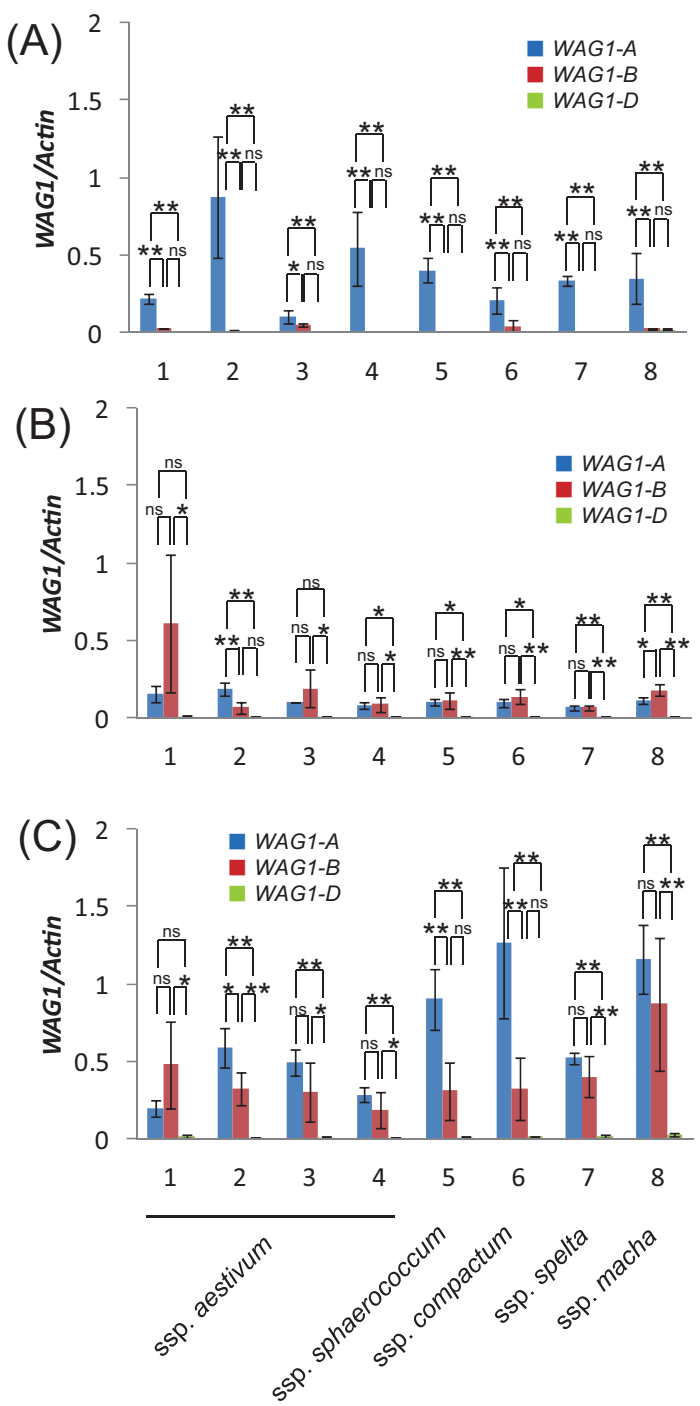

Fig. 4. Expression patterns of three WAG1 homoeologs examined by real-time PCR in hexaploid wheat using homoeologspecific primers for WAG1-A, WAG1-B and WAG1-D. Actin was used as the endogenous control. Total RNAs were isolated from (A) young spikes (3 to $10 \mathrm{~mm}$ in length), (B) stamens and (C) pistils of hexaploid species: (1) $T$. aestivum ssp. aestivum cv. Chinese Spring; (2) T. aestivum ssp. aestivum cv. Nanbukomugi; (3) T. aestivum ssp. aestivum (KU-11809); (4) T. aestivum ssp. aestivum (KU-1005); (5) T. aestivum ssp. sphaerococcum; (6) T. aestivum ssp. compactum; (7) T. aestivum ssp. spelta; (8) T. aestivum ssp. macha. Data represent means \pm standard errors from three replicate experiments. "ns" indicates no significant difference. Asterisk indicates a significant difference in a $t$-test (*, $P<0.05 ; * *, P<0.01)$.

with WAG1-D except in stamens of the bread wheat cv. Nanbukomugi (Fig. 4, B and C). At present, it is not clear why the expression level of WAG1-B in stamens of Nanbukomugi was so low compared to other varieties. WAG1-D was silenced or down-regulated in young spikes and reproductive floral organs (stamens and pistils) in all hexaploid wheat varieties, suggesting that suppression of the $\mathrm{D}$ genome homoeolog of WAG1 is a common feature of
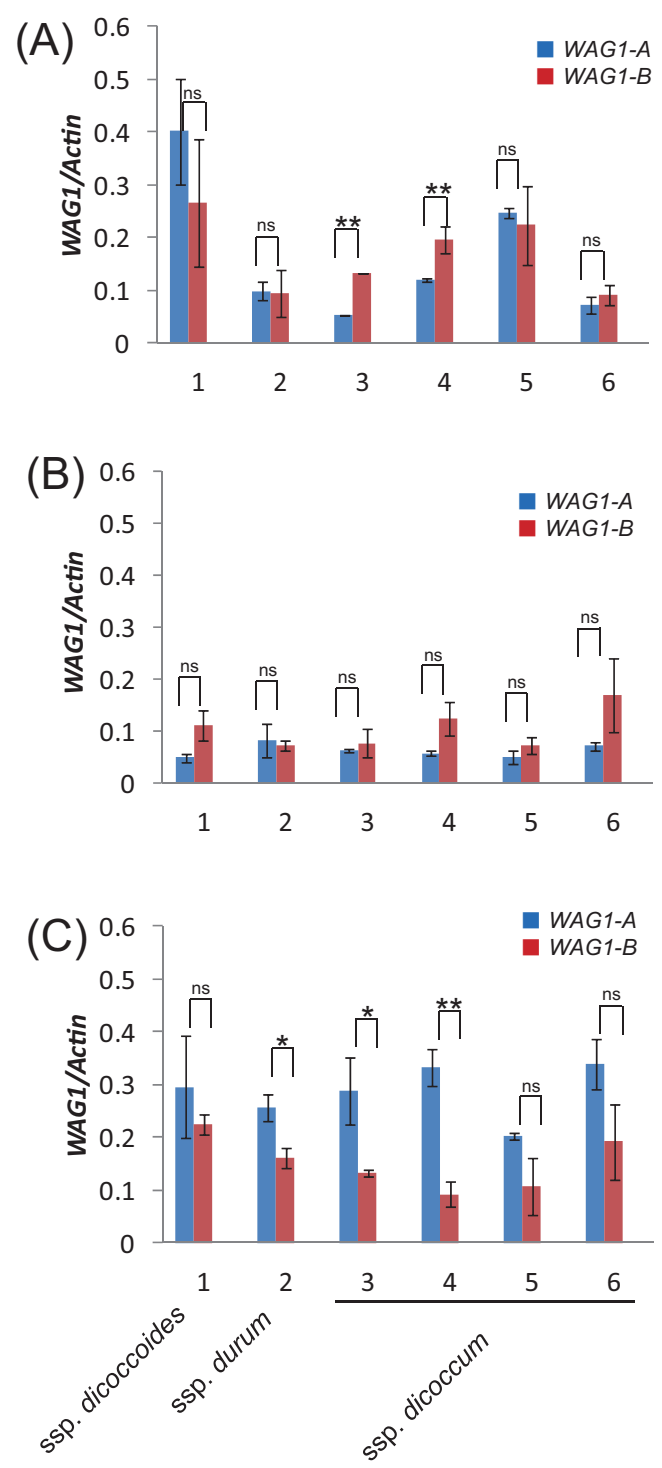

Fig. 5. Expression patterns of three WAG1 homoeologs examined by real-time PCR in tetraploid wheat using homoeologspecific primers for WAG1-A and WAG1-B. Actin was used as the endogenous control. Total RNAs were isolated from (A) young spikes (3 to $10 \mathrm{~mm}$ in length) (B) stamens and (C) pistils of tetraploid species: (1) T. turgidum ssp. dicoccoides; (2) $T$. turgidum ssp. durum; (3) T. turgidum ssp. dicoccon (KU-113); (4) T. turgidum ssp. dicoccon (KU-115); (5) T. turgidum ssp. dicoccon (KU-111); (6) T. turgidum ssp. dicoccon (KU-123). Data represent means \pm standard errors from three replicate experiments. "ns" indicates no significant difference. Asterisk indicates a significant difference in a $t$-test $\left(^{*}, P<0.05\right.$; **, $P<$ $0.01)$.

hexaploid wheat. Both WAG1-A and WAG1-B were expressed in young spikes, stamens and pistils of wild and cultivated tetraploid wheat lines (Fig. 5). This suggests that the down-regulation of WAG1-B in young spikes occurred during the allohexaploidization process between tetraploid wheat and Ae. tauschii. 
Expression pattern of class D gene WSTK in hexaploid and tetraploid wheat The levels of expression of WSTK homoeologs were determined in hexaploid wheat varieties (Fig. 6). In young spikes and pistils from all hexaploid wheat lines, WSTK-B was highly expressed but WSTK-D was significantly down-regulated and WSTK-A was silenced. In tetraploid wheat varieties, silencing of WSTK-A was observed in young spikes and very low expression was found in pistils (Fig. 7), suggesting that the suppression of WSTK-A occurred during allotetraploidization following the crossing of $\mathrm{A}$ and $\mathrm{B}$ genome donor species.

Expression pattern in synthetic hexaploid wheat lines To investigate the cause of the homoeolog-specific expression patterns, we examined WPI2, WAG1 and WSTK expression in four synthetic hexaploid wheat lines
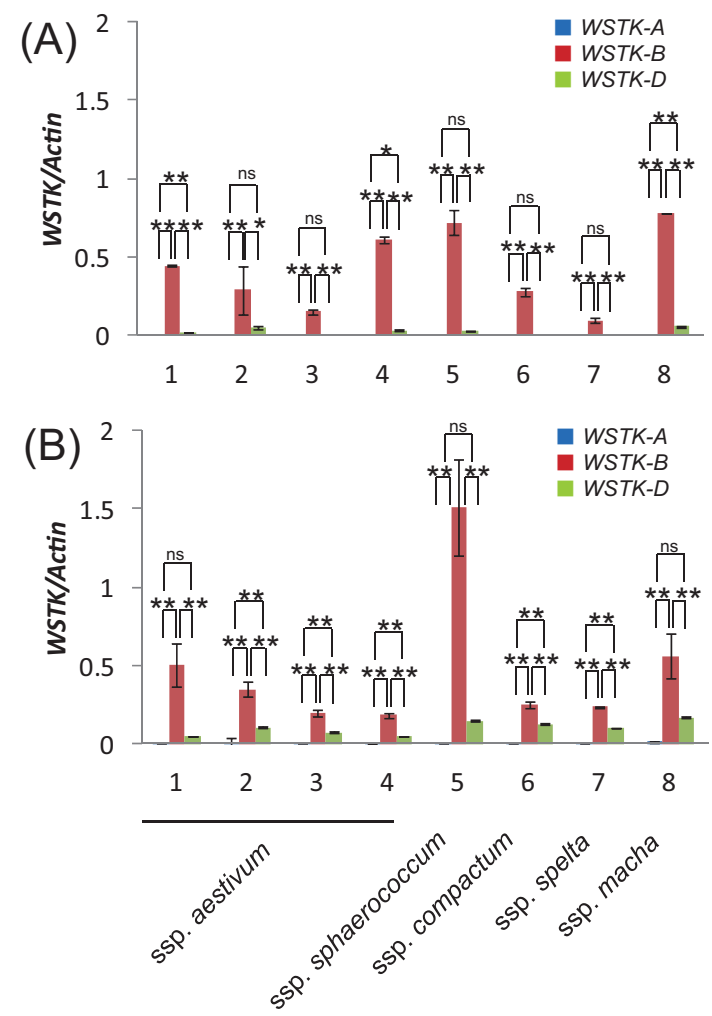

Fig. 6. Expression patterns of three WSTK homoeologs examined by real-time PCR in hexaploid wheat using homoeologspecific primers for WSTK-A, WSTK-B and WSTK-D. Actin was used as the endogenous control. Total RNAs were isolated from (A) young spikes (3 to $10 \mathrm{~mm}$ in length) and (B) pistils of hexaploid species: (1) T. aestivum ssp. aestivum cv. Chinese Spring; (2) T. aestivum ssp. aestivum cv. Nanbukomugi; (3) T. aestivum ssp. aestivum (KU-11809); (4) T. aestivum ssp. aestivum (KU-1005); (5) T. aestivum ssp. sphaerococcum; (6) T. aestivum ssp. compactum; (7) T. aestivum ssp. spelta; (8) T. aestivum ssp. macha. Data represent means \pm standard errors from three replicate experiments. " $n s$ " indicates no significant difference. Asterisk indicates a significant difference in a $t$-test (*, $P<0.05$; **, $P<0.01)$.
(Table 1). The synthetic hexaploid wheat lines were generated by crossing the tetraploid wheat $T$. turgidum (AABB) and diploid wheat Ae. tauschii (DD). Two types of synthetic hexaploid lines were used: first, previously generated synthetic hexaploids between $T$. turgidum ssp. dicoccoides or ssp. dicoccon and Ae. tauschii strains (old synthetic hexaploids) obtained from NBRP-KOMUGI; and second, newly generated synthetic hexaploids between T. turgidum ssp. durum cv. Langdon and Ae. tauschii strains (new synthetic hexaploids) generated by ourselves (Kajimura et al., 2011).

For the class B MADS-box gene WPI2, we found that homoeolog-specific expression patterns were similar among the four synthetic hexaploid wheat lines: WPI2-A and WPI2-D were expressed, while WPI2-B was downregulated in both young spikes and stamens except in the stamens of one of the old synthetic lines (\#1) (Fig. 8). This expression pattern is similar to that of natural hexaploid wheat varieties (Fig. 2). These results indicated that the homoeolog-specific expression patterns of
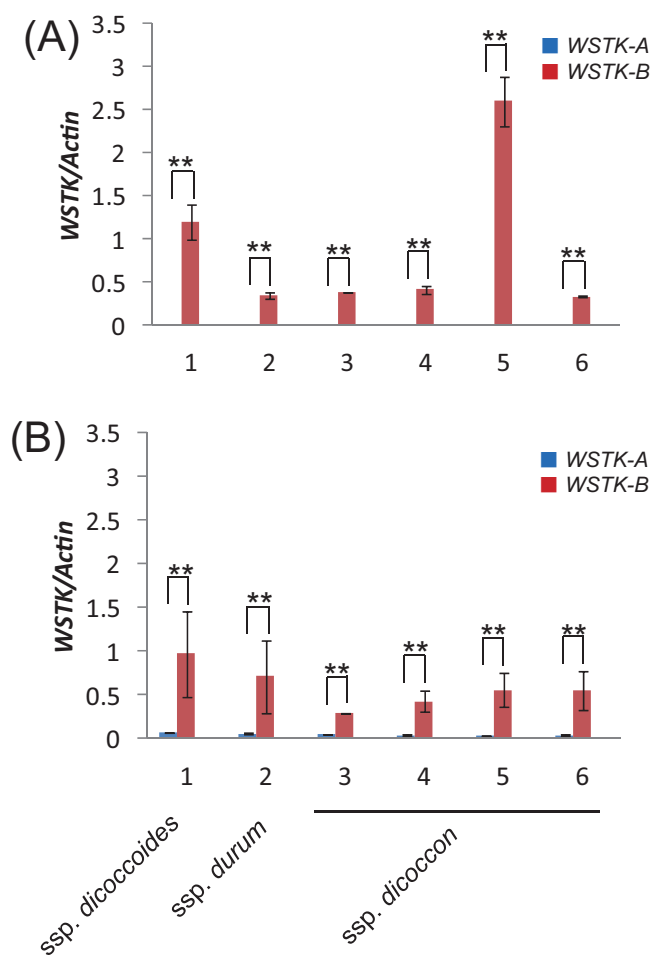

Fig. 7. Expression patterns of three WSTK homoeologs examined by real-time PCR in tetraploid wheat using homoeologspecific primers for WSTK-A and WSTK-B. Actin was used as the endogenous control. Total RNAs were isolated from (A) young spikes (3 to $10 \mathrm{~mm}$ in length) and (B) pistils of tetraploid species: (1) T. turgidum ssp. dicoccoides; (2) T. turgidum ssp. durum; (3) T. turgidum ssp. dicoccon (KU-113); (4) T. turgidum ssp. dicoccon (KU-115); (5) T. turgidum ssp. dicoccon (KU-111); (6) T. turgidum ssp. dicoccon (KU-123). Data represent means \pm standard errors from three replicate experiments. "ns" indicates no significant difference. Asterisk indicates a significant difference in a $t$-test $(*, P<0.05 ; * *, P<0.01)$. 


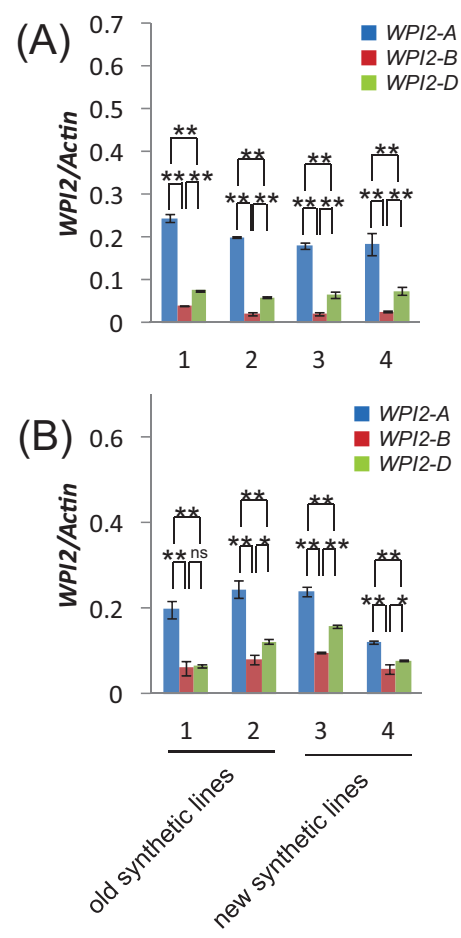

Fig. 8. Expression patterns of three WPI2 homoeologs examined by real-time PCR in synthetic hexaploid wheat using homoeolog-specific primers for WPI2-A, WPI2-B and WPI2-D. Actin was used as the endogenous control. Total RNAs were isolated from (A) young spikes (3 to $10 \mathrm{~mm}$ in length) and (B) stamens of synthetic hexaploid species: (1) T. turgidum ssp. dicoccoides var. spontaneonigrum KU-109 $\times$ Ae. tauschii ssp. strangulata KU-2074; (2) T. turgidum ssp. dicoccon cv. Vernal $\times$ Ae. tauschii; (3) T. turgidum ssp. durum cv. Langdon $\times$ Ae. tauschii PI476874; (4) T. turgidum ssp. durum cv. Langdon $\times$ Ae. tauschii CGN10768. Data represent means \pm standard errors from three replicate experiments. "ns" indicates no significant difference. Asterisk indicates a significant difference in a $t$-test (*, $P<0.05$; **, $P<0.01)$.

WPI2 were a consistent and reproducible feature of hexaploid lines.

For the class C MADS-box gene WAG1, we found high expression of WAG1-A, very low expression of WAG1-B and silencing of WAG1-D in young spikes (Fig. 9A). WAG1-A and WAG1-B were expressed in stamens and pistils, but WAG1-D showed silencing or a very low expression level (Fig. 9, B and C). These homoeologspecific expression patterns in different organs were similar to those of natural hexaploid wheat varieties (Fig. 4) and indicate that expression patterns of WAG1 homoeologs were a consistent and reproducible feature of hexaploid lines.

For the class D MADS-box gene WSTK1, only WSTK-B was preferentially expressed in both young spikes and pistils (Fig. 10). This expression pattern was similar to that in natural hexaploid wheat lines (Fig. 6) and indicates that expression patterns of WSTK homoeologs were a consistent and reproducible feature of hexaploid lines.
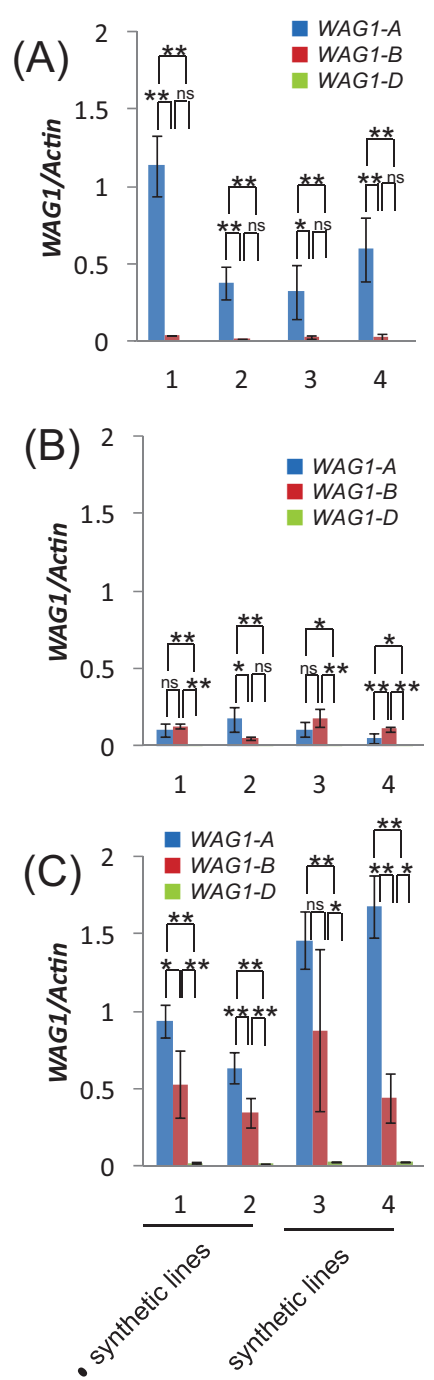

Fig. 9. Expression patterns of three WAG1 homoeologs examined by real-time PCR in synthetic hexaploid wheat using homoeolog-specific primers for WAG1-A, WAG1-B and WAG1-D. Actin was used as the endogenous control. Total RNAs were isolated from (A) young spikes (3 to $10 \mathrm{~mm}$ in length), (B) stamens and (C) pistils of synthetic hexaploid lines: (1) T. turgidum ssp. dicoccoides var. spontaneonigrum KU-109 $\times$ Ae. tauschii ssp. strangulata KU-2074; (2) T. turgidum ssp. dicoccon cv. Vernal $\times$ Ae. tauschii; (3) T. turgidum ssp. durum cv. Langdon $\times$ Ae. tauschii PI476874; (4) T. turgidum ssp. durum cv. Langdon $\times A e$. tauschii CGN10768. Data represent means \pm standard errors from three replicate experiments. "ns" indicates no significant difference. Asterisk indicates a significant difference in a $t$-test (*, $P<0.05$; ** $P<0.01$ ).

\section{DISCUSSION}

Allopolyploidization, the doubling of chromosome number in species hybrids, generates polyploids with genome sets derived from related species. For example, hexaploid bread wheat has the AABBDD genome composed of genomic contributions from three different ancestral species. Allopolyploidization is an important process 

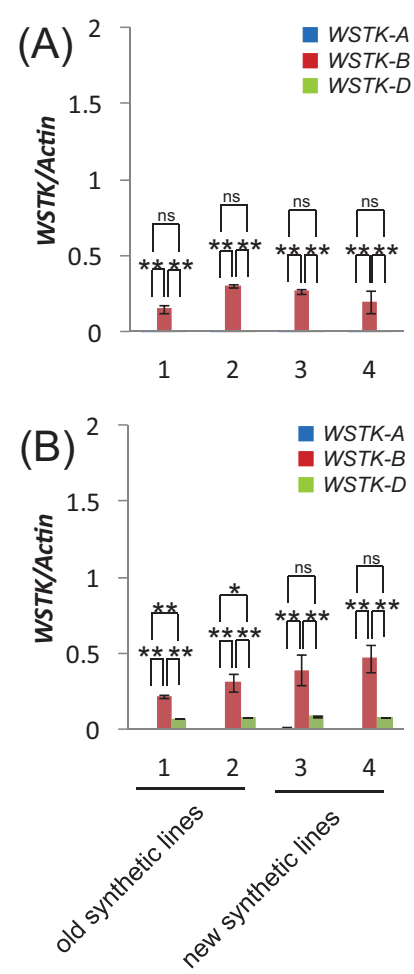

Fig. 10. Expression patterns of three WSTK homoeologs examined by real-time PCR in synthetic hexaploid wheat using homoeolog-specific primers for WSTK-A, WSTK-B and WSTK-D. Actin was used as the endogenous control. Total RNAs were isolated from (A) young spikes (3 to $10 \mathrm{~mm}$ in length) and (B) pistils of synthetic hexaploid species: (1) T. turgidum ssp. dicoccoides var. spontaneonigrum KU-109 $\times$ Ae. tauschii ssp. strangulata KU-2074; (2) T. turgidum ssp. dicoccon cv. Vernal $\times$ Ae. tauschii; (3) T. turgidum ssp. durum cv. Langdon $\times$ Ae. tauschii PI476874; (4) T. turgidum ssp. durum cv. Langdon $\times A e$. tauschii CGN10768. Data represent means \pm standard errors from three replicate experiments. "ns" indicates no significant difference. Asterisk indicates a significant difference in a $t$-test (*, $P<0.05 ; * *, P<0.01)$.

both for angiosperm evolution and for functional innovations, such as the development of inflorescence structure (Shitsukawa et al., 2009). The polyploid genome displays dynamic changes in gene expression patterns caused by so-called "genomic shock" (McClintock, 1984). Feldman and Levy (2005) suggested that this phenomenon might induce rapid genomic changes (revolutionary change), through either genetic or epigenetic alterations, over a comparatively short period during allopolyploidization. These changes could include: (1) non-random elimination of coding and non-coding DNA; (2) epigenetic changes of coding and non-coding DNA; and (3) activation of genes and retroelements induced by alteration of adjacent DNA sequences. Recently, studies on the molecular pathways affected by polyploidization have greatly progressed. In particular, the genetic diversification and epigenetic modifications of transcription factors have been shown to influence many steps in the intricate and coordinately regulated biological pathways of allopolyploid species (Chen, 2007).

The expression profiles of homoeologous genes have previously been investigated in hexaploid wheat using the frequencies of ESTs (Mochida et al., 2003). This study indicated that expression patterns could be classified into two major groups: genes that are almost equally expressed from all three genomes; and genes that are expressed differentially in the three genomes, and which vary between tissues. Subsequently, the differential expression of homoeologs was measured using cDNASSCPs and microarray analysis (Pumphrey et al., 2009), while more recently, genome-wide gene expression changes during allopolyploidization were investigated using Affymetrix GeneChip Wheat Genome Arrays (Changué et al., 2010; Akhunova et al., 2010; Qi et al., 2012) and an Agilent 38K oligo-DNA microarray (Jung et al., 2014). These studies indicate that up to $20 \%$ of genes show non-additive expression with homoeolog-specific upor down-regulation. Leach et al. (2014) performed RNAseq analysis using next generation sequencing with nullisomic-tetrasomic lines of chromosome groups 1 and 5 in hexaploid wheat to identify homoeolog-specific expression patterns. They assessed 2354 genes from homoeologous chromosomes and showed that at least $45 \%$ are expressed from all three homoeologs, but that most of the genes have strikingly biased expression patterns among the homoeologs. The remaining $55 \%$ of genes are expressed from one or two homoeologs only. These observations indicate that differentiation of expression among homoeologs is common in the hexaploid wheat genome.

In order to understand the meaning of homoeologspecific expression differences, we have started to study functional differentiation in genes involved in floral organ formation. Ultimately, our goal is to elucidate the role of homoeolog-specific expression of MADS-box genes in this developmental pathway. This will also involve determining the function of homoeolog-specific protein-protein interactions. As a first step in this investigation, we examined homoeolog-specific expression patterns of class $\mathrm{B}, \mathrm{C}$ and D MADS-box genes in floral development in allopolyploid wheat.

\section{Homoeolog-specific expression patterns varies} among MADS-box genes Our quantitative real-time PCR analysis showed that in young spikes of hexaploid wheat, the class B WPI2 genes of the A and D genomes (WPI2-A and WPI2-D) were preferentially expressed (Fig. 2A), the class C WAG1 gene in the A genome (WAG1-A) was preferentially expressed (Fig. $4 \mathrm{~A}$ ), and the class D WSTK gene of the B genome (WSTK-B) was preferentially expressed (Fig. 6A). Our previous study revealed that class E MADS-box gene WSEP homoeologs did not show any change in expression patterns and protein functions 
(Shitsukawa et al., 2007). However, only the D genome copy of WLHS1, another class $\mathrm{E}$ gene, functions in hexaploid wheat. These results indicate that the homoeologs of each MADS-box gene for floral organ development show a specific differential expression pattern.

The concept of 'genomic expression dominance' has been developed in allotetraploid cotton; in this species, homoeolog expression is biased in favor of the $\mathrm{D}$ genome over the A genome (Rapp et al., 2009; Flagel and Wendel, 2010). Genomic expression dominance has also been reported in synthetic hexaploid wheat which shows a biased contribution from the $\mathrm{AB}$ genome of the tetraploid parent (Akhunova et al., 2010). The AB genome homoeologs involved in protein biosynthesis and photosynthesis were up-regulated, suggesting that they may contribute to restoration of growth vigor in allopolyploid wheat. The present study is the first to demonstrate that there is no common genomic expression dominance mechanism among floral MADS-box genes in hexaploid wheat, but rather that the pattern of differential expression of homoeologs varies among the genes (Figs. 2, 4, and 6). Future studies will be need to elucidate the common features of the gene systems involved in developmental pathways such as flower development in allopolyploid plants.

Homoeolog-specific expression patterns are consistently, reproducibly and rapidly established during allopolyploidization The homoeolog-specific expression patterns of class B, C, D MADS-box genes were consistent in all hexaploid wheat lines studied, including synthetic hexaploid wheat lines (Figs. 2, 4, 6, 8, 9 and 10), suggesting that these patterns of floral MADS-box gene expression are not stochastically determined but are controlled by an as yet unidentified mechanism that develops during allopolyploidization. Bottley and Koebner (2008) examined the silencing of 15 EST homoeologs in 16 modern northern European winter wheat varieties by SSCP analysis. The homoeolog-specific silencing patterns differed among varieties, and only two varieties shared the same pattern. More recently, Qi et al. (2012) compared homoeolog-specific expression patterns among two newly synthesized hexaploid wheat lines based on data from Affymetrix GeneChip Wheat Genome Arrays. This analysis showed that only 27 of 3539 genes had the same homoeolog-specific expression pattern in two synthetic hexaploid lines. Taken together with the present study, a unique feature of floral MADS-box genes has been revealed, namely, that the homoeolog-specific expression patterns are determined in a consistent manner among different polyploid varieties.

In young spikes of tetraploid wheat, WPI2- $B$ was more highly expressed than WPI2-A (Fig. 3A). However, the expression of WPI2-B was significantly down-regulated compared to that of WPI2-A, which was up-regulated in hexaploid wheat (Fig. 2A). Newly synthesized hexaploid wheat lines showed the same pattern of down-regulation of WPI2-B as natural hexaploid wheat varieties (Fig. $8 \mathrm{~A})$. These results suggest that homoeolog-specific down-regulation in young spikes occurred early in allohexaploidization of tetraploid wheat and Ae. tauschii hybrids. We also found that WAG1-B was significantly down-regulated in young spikes of hexaploid wheat varieties (Fig. 4A), while WAG1-B was expressed in the young spikes of tetraploid varieties (Fig. 5A). Synthetic hexaploid lines showed down-regulation of WAG1-B similar to natural hexaploid wheat varieties (Fig. 9A). These results suggest that the down-regulation of WAG1-B in young spikes occurred early in allohexaploidization between tetraploid wheat and Ae. tauschii. In summary, as newly generated synthetic hexaploid wheat lines showed similar homoeolog-specific expression patterns for floral MADS-box genes as natural hexaploid wheat varieties, then this control mechanism must develop relatively rapidly and reproducibly at an early stage in the generation of allopolyploids.

One of the causes of non-additive expression associated with homoeolog-specific up- or down-regulation is the trans-regulation of regulatory factor(s) and target genes located on different genomes in allopolyploid species (Soltis et al., 2014). In diploid species, only cisregulation occurs between regulatory factor(s) and target genes. Complicated trans-regulation together with cisregulation could be involved in homoeolog expression in allopolyploids, leading to the homoeolog-specific nonadditive expression patterns.

It is also known that epigenetic alterations accompany allopolyploidization and can, occasionally, lead to gene silencing. Such epigenetic alterations have been reported in polyploids of Arabidopsis (Lee and Chen, 2001; Wang et al., 2004), Triticum (Shitsukawa et al., 2007; Zhao, N. et al., 2011), and Brassica (Lukens et al., 2006). Therefore, it will be important to determine in future studies whether homoeolog-specific expression patterns in floral MADS-box genes are regulated by an epigenetic mechanism. A recent RNA-seq analysis in synthetic hexaploid wheat suggested that a mechanism involving small RNAs may mediate dynamic homoeolog regulation $(\mathrm{Li}$ et al., 2014). Identification of micro RNAs and small interfering RNAs that target floral MADS-box genes would be a further step in elucidating the molecular mechanism of homoeolog-specific expression.

Organ-specific patterning of homoeolog-specific expression We found that WAG1-B was significantly down-regulated in young spikes, but was expressed in stamens and pistils of hexaploid wheat varieties (Fig. 4). Furthermore, in tetraploid wheat varieties, WPI2-A was more weakly expressed than WPI2- $B$ in young spikes, while WPI2-A was more highly expressed than WPI2-B in 
stamens (Fig. 3). An SSCP analysis identified organspecific partitioning of the expression levels of homoeologs of Alcohol dehydrogenase $A(A d h A)$ in cotton flowers (Adams et al., 2003). In this case, both $A d h A$ homoeologs were expressed in vegetative tissues, but one copy was predominantly expressed in petals and stamens while the other copy was more higher expressed in carpels. Another SSCP analysis found organ-specific silencing of homoeologs in hexaploid wheat (Bottley et al., 2006). Such functional differentiation is likely to have occurred at an early stage in allopolyploidization (Adams et al., 2004). The differences in homoeolog-specific expression levels in different tissues and organs suggest that the MADS-box gene homoeologs are functionally divergent in floral development after allopolyploidization.

\section{CONCLUSION}

The present study identified homoeolog-specific expression patterns in floral MADS-box genes in allopolyploid wheat and showed: 1) the homoeolog-specific expression pattern was different in each MADS-box gene; 2) the homoeolog-specific expression patterns were consistently, reproducibly and rapidly established during allopolyploidization; and 3) organ-specific expression changes occurred among homoeologs in some MADS-box genes. The floral homeotic MADS domain proteins interact in floral tissues as proposed in the "floral quartet" model; in this model, a tetramer of MADS-domain proteins functions in the specification of floral organ identity (Smaczniak et al., 2012). The amino acid sequences of WPI2, WAG1 and WSTK differ among homoeologs, suggesting that the complex protein-protein interactions of homoeologs of MADS-box genes are probably associated with morphological and physiological diversification in floral development in wheat plants with different ploidy levels.

We are grateful to the National Bioresource Project - Wheat (NBRP-KOMUGI) for providing wheat materials and the EST database. This work was supported in part by a Grant-in-Aid for Challenging Exploratory Research from the Ministry of Education, Culture, Sports, Science and Technology of Japan (No. 24658008) to K. Murai.

\section{REFERENCES}

Adams, K. L., Cronn, R., Percifield, R., and Wendel, J. F. (2003) Genes duplicated by polyploidy show unequal contributions to the transcriptome and organ-specific reciprocal silencing. Proc. Natl. Acad. Sci. USA 100, 4649-4654.

Adams, K. L., Percifield, R., and Wendel, J. F. (2004) Organspecific silencing of duplicated genes in a newly synthesized cotton allotetraploid. Genetics 168, 2217-2226.

Akhunova, A. R., Matniyazov, R. T., Liang, H., and Akhunov, E. D. (2010) Homoeolog-specific transcriptional bias in allopo- lyploid wheat. BMC Genomics 11, 505.

Bottley, A., and Koebner, R. M. D. (2008) Variation for homoeologous gene silencing in hexaploid wheat. Plant J. 56, 297302.

Bottley, A., Xia, G. M., and Koebner, R. M. D. (2006) Homoeologous gene silencing in hexaploid wheat. Plant J. 47, 897906.

Changué, V., Just, J., Mestiri, I., Balzergue, S., Tanguy, A.-M., Huneau, C., Huteau, V., Belcram, H., Coriton, O., Jahier, J., et al. (2010) Genome-wide gene expression changes in genetically stable synthetic and natural wheat allohexaploids. New Phytologist 187, 1181-1194.

Chen, Z. J. (2007) Genetic and epigenetic mechanisms for gene expression and phenotypic variation in plant polyploids. Ann. Rev. Plant Biol. 58, 377-406.

Ditta, G., Pinyopich, A., Robles, P., Pelaz, S., and Yanofsky, M. F. (2004) The SEP4 gene of Arabidopsis thaliana functions in floral organ and meristem identity. Curr. Biol. 14, 1935-1940.

Favaro, R., Pinyopich, A., Battaglia, R., Kooiker, M., Borghi, L., Ditta, G., Yanofsky, M. F., Kater, M. M., and Colombo, L. (2003) MADS-box protein complexes control carpel and ovule development in Arabidopsis. Plant Cell 15, 26032611.

Feldman, M. (2001) The origin of cultivated wheat. In: The World Wheat Book: A History of Wheat Breeding. (eds.: Bonjean, A. P., and Angus, W. J.), pp. 1-56. Lavoisier Publishing, Londres.

Feldman, M., and Levy, A. A. (2005) Allopolyploidy - a shaping force in the evolution of wheat genomes. Cytogenet. Genome Res. 109, 250-258.

Flagel, L. E., and Wendel, J. F. (2010) Evolutionary rate variation, genomic dominance and duplicate gene expression evolution during allotetraploid cotton speciation. New Phytologist 186, 184-193.

Goto, K., and Meyerowitz, E. M. (1994) Function and regulation of the Arabidopsis floral homeotic gene PISTILLATA. Genes Dev. 8, 1548-1560.

Hama, E., Takumi, S., Ogihara, Y., and Murai, K. (2004) Pistillody is caused by alterations to the class-B MADS-box gene expression pattern in alloplasmic wheats. Planta 218 , $712-720$.

Hirabayashi, C., and Murai, K. (2009) Class C MADS-box gene AGAMOUS was duplicated in the wheat genome. Wheat Inf. Serv. 107, 13-16.

Jack, T., Brockman, L. L., and Meyerowitz, E. M. (1992) The homeotic gene APETALA3 of Arabidopsis thaliana encodes a MADS box and is expressed in petals and stamens. Cell 68, 683-697.

Jung, Y., Kawaura, K., Mishina, K., Sakuma, S., Kishii, M., and Ogihara, Y. (2014) Changes in genome-wide gene expression during allopolyploidization and genome stabilization in hexaploid wheat. Genes Genet. Syst. 89, 215-225.

Kajimura, T., Murai, K., and Takumi, S. (2011) Distinct genetic regulation of flowering time and grain-filling period based on empirical study of D-genome diversity in synthetic hexaploid wheat lines. Breed. Sci. 61, 130-141.

Kinjo, H., Shitsukawa, N., Takumi, S., and Murai, K. (2012) Diversification of three APETALA1 / FRUITFULL-like genes in wheat. Mol. Genet. Genomics 287, 283-294.

Leach, L. J., Belfield, E. J., Jiang, C., Brown, C., Mithani, A., and Harberd, N. P. (2014) Patterns of homoeologous gene expression shown by RNA sequencing in hexaploid bread wheat. BMC Genomics 15, 276.

Lee, H.-S., and Chen, Z. J. (2001) Protein-coding genes are epi- 
genetically regulated in Arabidopsis polyploids. Proc. Natl. Acad. Sci. USA 98, 6753-6758.

Li, A., Liu, D., Wu, J., Zhao, X., Hao, M., Geng, S., Yan, J., Jiang, X., Zhang, L., Wu, J., et al. (2014) mRNA and small RNA transcriptomes reveal insights into dynamic homoeolog regulation of allopolyploid heterosis in nascent hexaploid wheat. Plant Cell 26, 1878-1900.

Lukens, L. N., Pires, J. C., Leon, E., Vogelzang, R., Oslach, L., and Osborn, T. (2006) Patterns of sequence loss and cytosine methylation within a population of newly resynthesized Brassica napus allopolyploids. Plant Physiol. 140, 336348.

Mandel, M. A., Gustafson-Brown, C., Savidge, B., and Yanofsky, M. F. (1992) Molecular characterization of the Arabidopsis floral homeotic gene APETALA1. Nature 360, 273-277.

McClintock, B. (1984) The significance of responses of the genome to challenge. Science 226, 792-801.

Meguro, A., Takumi, S., Ogihara, Y., and Murai, K. (2003) WAG, a wheat AGAMOUS homolog, is associated with development of pistil-like stamens in alloplasmic wheats. Sex. Plant Reprod. 15, 221-230.

Mochida, K., Yamazaki, Y., and Ogihara, Y. (2003) Discrimination of homoeologous gene expression in hexaploid wheat by SNP analysis of contigs grouped from a large number of expressed sequence tags. Mol. Genet. Genomics 270, 371377.

Mochida, K., Kawaura, K., Shimosaka, E., Kawakami, N., ShinI, T., Kohara, Y., Yamazaki, Y., and Ogihara, Y. (2006) Tissue expression map of a large number of expressed sequence tags and its application to in silico screening of stress response genes in common wheat. Mol. Genet. Genomics 276, 304-312.

Murai, K. (2013) Homeotic genes and the ABCDE model for floral organ formation in wheat. Plants 2, 379-395.

Paolacci, A. R., Tanzarella, O. A., Porceddu, E., Varotto, S., and Ciaffi, M. (2007) Molecular and phylogenetic analysis of MADS-box genes of MIKC type and chromosome location of $S E P$-like gene in wheat (Triticum aestivum L.). Mol. Genet. Genomics 278, 689-708.

Pelaz, S., Ditta, G. S., Baumann, E., Wisman, E., and Yanofsky, M. F. (2000) B and C floral organ identity functions require SEPALLATA MADS-box genes. Nature 405, 200-203.

Pinyopich, A., Ditta, G. S., Savidge, B., Liljegren, S. J., Baumann, E., Wisman, E., and Yanofsky, M. F. (2003) Assessing the redundancy of MADS-box genes during carpel and ovule development. Nature 424, 85-88.

Pumphrey, M., Bai, J., Laudencia-Chingcuanco, D., Anderson, O., and Gill, B. S. (2009) Nonadditive expression of homoeologous genes is established upon polyploidization in hexaploid wheat. Genetics 181, 1147-1157.

Qi, B., Huang, W., Zhu, B., Zhong, X., Guo, J., Zhao, N., Xu, C.,
Zhang, H., Pang, J., Han, F., et al. (2012) Global transgenerational gene expression dynamics in two newly synthesized allohexaploid wheat (Triticum aestivum) lines. BMC Biol. 10, 3.

Rapp, R. A., Udall, J. A., and Wendel, J. F. (2009) Genomic expression dominance in allopolyploids. BMC Biol. 7, 18.

Rijpkema, A. S., Vandenbussche, M., Koes, R., Heijmans, K., and Gerats, T. (2010) Variations on a theme: Changes in the floral ABCs in angiosperms. Semin. Cell Dev. Biol. 21, 100-107.

Shitsukawa, N., Tahira, C., Kassai, K., Hirabayashi, C., Shimizu, T., Takumi, S., Mochida, K., Kawaura, K., Ogihara, Y., and Murai, K. (2007) Genetic and epigenetic alteration among three homoeologous genes of a class E MADS box gene in hexaploid wheat. Plant Cell 19, 1723-1737.

Shitsukawa, N., Kinjo, H., Takumi, S., and Murai, K. (2009) Heterochronic development of the floret meristem determines grain number per spikelet in diploid, tetraploid and hexaploid wheats. Ann. Botany 104, 243-251.

Smaczniak, C., Immink, R. G. H., Angenent, G. C., and Kaufmann, K. (2012) Developmental and evolutionary diversity of plant MADS-domain factors: Insights from recent studies. Development 139, 3081-3098.

Soltis, D. E., Visger, C. J., and Soltis, P. S. (2014) The polyploidy revolution then... and now: Stebbins revisited. Am. J. Bot. 101, 1057-1078.

Tsunewaki, K. (1980) Genetic diversity of the cytoplasm in Triticum and Aegilops. Japan Society for the Promotion of Science, Tokyo, Japan.

Wang, J., Tian, L., Madlung, A., Lee, H.-S., Chen, M., Lee, J. J., Watson, B., Kagochi, T., Comai, L., and Chen, Z. J. (2004) Stochastic and epigenetic changes of gene expression in Arabidopsis polyploids. Genetics 167, 1961-1973.

Yamada, K., Saraike, T., Shitsukawa, N., Hirabayashi, C., Takumi, S., and Murai, K. (2009) Class D and B $_{\text {sister }}$ MADSbox genes are associated with ectopic ovule formation in the pistil-like stamens of alloplasmic wheat (Triticum aestivum L.). Plant Mol. Biol. 71, 1-14.

Yanofsky, M. F., Ma, H., Bowman, J. L., Drews, G. N., Feldmann, K. A., and Meyerowitz, E. M. (1990) The protein encodes by the Arabidopsis homeotic gene agamous resembles transcription factors. Nature 346, 35-39.

Zhao, N., Zhu, B., Li, M., Wang, L., Xu, L., Zhang, H., Zheng, S., Qi, B., Han, F., and Liu, B. (2011) Extensive and heritable epigenetic remodeling and genetic stability accompany allohexaploidization of wheat. Genetics 188, 499-509.

Zhao, T., Ni, Z., Dai, Y., Yao, Y., Nie, X., and Sun, Q. (2006) Characterization and expression of 42 MADS-box genes in wheat (Triticum aestivum L.). Mol. Genet. Genomics 276, 334-350. 